Dear Author,

Please, note that changes made to the HTML content will be added to the article before publication, but are not reflected in this PDF.

Note also that this file should not be used for submitting corrections. 


\section{Hybrid 2D-nanomaterials-based electrochemical immunosensing strategies for clinical biomarkers determination}

\begin{abstract}
A B S T R A C T
Owing to the outstanding conductivity and biocompatibility as well as numerous other fascinating properties of two-dimensional (2D)-nanomaterials, 2D-based nanohybrids have shown unparalleled superiorities in the field of electrochemical biosensors. This review highlights latest advances in electrochemical immunosensors for clinical biomarkers based on different hybrid 2D-nanomaterials. Particular attention will be given to hybrid nanostructures involving graphene and other graphene-like 2Dlayered nanomaterials (GLNs). Several recent strategies for using such 2D-nanomaterial heterostructures in the development of modern immunosensors, both for tagging or modifying electrode transducers, are summarized and discussed. These hybrid nanocomposites, quite superior than their rival materials, will undoubtedly have an important impact within the near future and not only in clinical areas. Current challenges and future perspectives in this rapidly growing field are also outlined.
\end{abstract}

c) 2016 Published by Elsevier B.V.

\section{Contents}

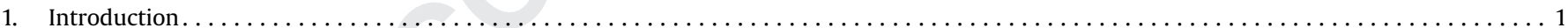

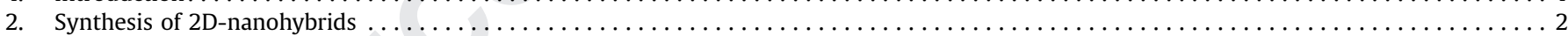

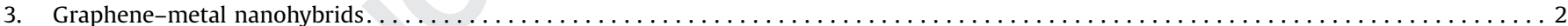

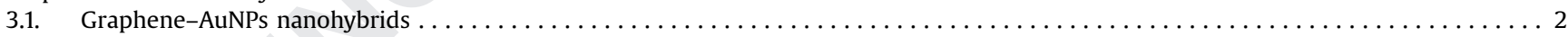

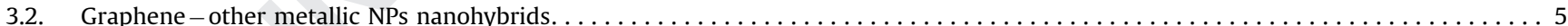

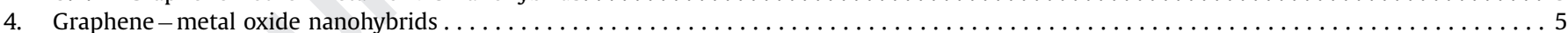

4.1. Graphene-metal oxide nanoparticles hybrids as electrode surface modifiers in electrochemical immunosensors.............. 5

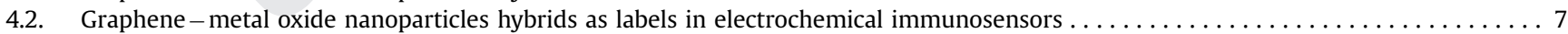

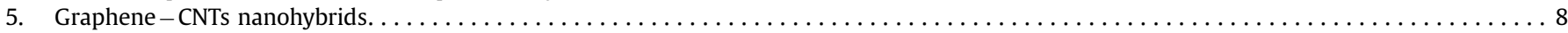

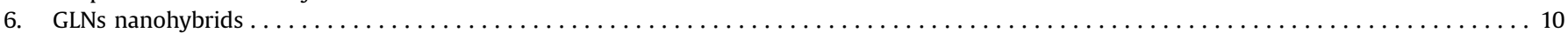

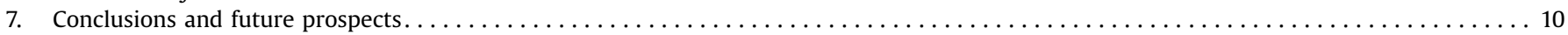

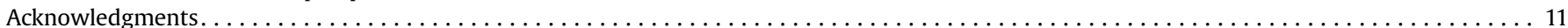

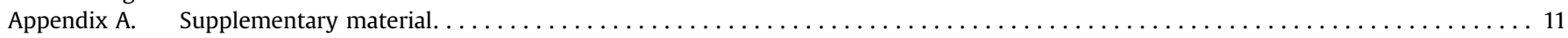

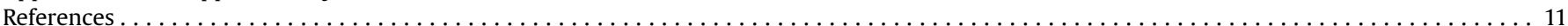

\section{Introduction}

A key to develop nanodevices with high performance for

\footnotetext{
* Corresponding author.

E-mail addresses: pingarro@quim.ucm.es (G.-P. Nikoleli), pingarro@quim.ucm.es (J.M. Pingarrón).
}

http://dx.doi.org/10.1016/j.bios.2016.01.042

0956-5663/৫ 2016 Published by Elsevier B.V. biosensors applications is to explore advanced functional nanomaterials (Yang et al., 2015). Recently, 2D-layered nanomaterials have aroused tremendous interest due to their promising properties and a broad range of applications in electronics, optoelectronics, catalysis, and energy storage devices (Su et al., 2015). Among them, graphene ( $\mathrm{Gr}$ ) has emerged as the most powerful 2D-nanomaterial in developing electrochemical sensors ( $\mathrm{Su}$ et al., 
2015; Kumar et al., 2015; Song et al., 2016). It is a 2D-single atomic planar sheet of $\mathrm{sp}^{2}$ bonded carbon atoms densely packaged into a honeycomb lattice structure (Brownson and Banks, 2010), exhibiting one of the highest mechanical strength among all materials, extraordinary electron transfer capability and excellent thermal conductivity (Song et al., 2016). Properties such as excellent conductivity while electronically being a low-noise material, wide potential window, exceptional biocompatibility, chemical inertness, high specific surface area and easy functionalization, mass production and suitability for various modes of sensing and detection, endow graphene and its related composites and hybrids with great applicability in the field of electrochemical biosensors (Gan and Zhao, 2015).

A crucial part in biosensing is to properly immobilize the bioreceptor or analyte on the electrode surface. Graphene, not only possesses a large specific surface area with high capacity to accumulate analyte molecules for high sensitive detection, but also exhibits a unique structure: cavities in the wall surface and tunable morphologies and compositions, which are particularly advantageous for electroanalysis. Moreover, the surfaces of graphene derivatives are rich in functional groups which offer versatile approaches to couple molecular receptors for electrochemical sensing (Su et al., 2015). Thus, graphene has been widely used for the immobilization of a series of recognition probes and for the modification of biosensing interfaces with controlled unique structures, which have shown, in particular, a promising performance in biomedical analysis (Song et al., 2016).

The widespread investigation of graphene has also drawn a current surge of interest in other 2D-nanomaterials. Graphene-like 2D-layered nanomaterials (GLNs) including, among others, boron nitride (BN) nanosheets and transition metal dichalcogenides (TMDs, e.g. $\mathrm{MoS}_{2}$ and $\mathrm{WS}_{2}$ ) show also strong mechanical strength, high surface area, unparalleled thermal conductivity, remarkable biocompatibility and ease of functionalization, and accordingly they have attracted also significant interest in the development of electrochemical (bio)sensors (Pumera and Loo, 2014; Lee et al., 2014; Huang et al., 2014; Yang et al., 2015; Su et al., 2015; Kumar et al., 2015).

In addition, hybrid nanostructures can be defined as materials composed of two or several components with at least one of them owning a dimension in the nanoscale regime. They possess the advantages of individual components but also may exhibit new properties and functions for practical applications (Huang et al., 2014).

Although graphene exhibits unique 2-D structural, chemical, and electronic properties, these only emerge in the 2D planar direction limiting its scope and application (Badhulika et al., 2015). Moreover, some inherent disadvantages of pristine graphene, such as easy aggregation, poor solubility and/or processability, represent significant obstacles in the development of electrochemical biosensors (Gan and Zhao, 2015; Li et al., 2015). Therefore, to optimize and further expand the use of this 2D-nanomaterial in electrochemistry, new efforts in graphene research have attempted to address these weaknesses by developing structures wherein graphene acts as a scaffold for anchoring other nanomaterials. These graphene hybrids, apart from taking full advantage of the superior properties of graphene and the corresponding functionalizing nanomaterial, are also endowed with new desirable properties (Gan and Zhao, 2015). In fact, hybrids comprised of 2Dnanomaterials can function as novel electrode materials, signal amplifiers, and catalysts of the electrochemical reaction of the in situ generated product during the biosensing event. To date, the most common hybrid 2D-nanomaterials applicable to electrochemical biosensing include graphene-carbon nanotubes $(\mathrm{Gr}-\mathrm{CNT})$, graphene - semiconductor nanomaterials and graphene-metal nanomaterial hybrids, constituting a widely emerging field of research (Badhulika et al., 2015). The recently successful synthesis of graphene via various protocols and the integration of graphene with different nanomaterials, such as metals, metal oxides, and quantum dots (QDs) can provide abundant opportunities for developing novel biosensors with enhanced performance (Mao et al., 2013; Song et al., 2016).

Given the high interest and wide scope of this type of hybrid nanomaterials, this review discusses recent advances in the field of electrochemical immunosensing platforms based on hybrid 2Dnanomaterials for clinical biomarkers, classifying the highlighted approaches according to the type of hybrid 2D-nanomaterial used, as well as its use as electrode modifiers or nanocarriers of signal molecules. In addition, the bottlenecks involved and possible research directions are also pointed out.

\section{Synthesis of 2D-nanohybrids}

Generally, there are three ways to synthesize hybrid nanostructures based on 2D-nanomaterials: i) controlled growth of other functional materials on 2D-nanomaterials as synthetic templates, ii) growth and/or assembly of 2D-nanomaterials on 1Dand 3D-templating structures to form porous hierarchical architectures, and iii) formation of heterostructures by stacking different kinds of 2D-materials to artificially create van der Waals layered crystals (Huang et al., 2014).

A number of methods have been developed to prepare these 2D-layered materials involving top-down and bottom-up approaches, such as chemical vapor deposition (CVD), epitaxial growth, liquid, mechanical and electrochemical exfoliation, lithium assisted intercalation and exfoliation, direct sonication in solvents, solvothermal/hydrothermal methods and microwave synthesis (Huang et al., 2014; Kumar et al., 2015; Yang et al., 2015). In addition to these methodologies, graphene can be prepared by reduction of graphene oxide (GO) and longitudinal 'unzipping' of carbon nanotubes (CNTs) (Li et al., 2015). More information about the synthesis of 2D-nanohybrids can be found in Supplementary Information.

\section{Graphene-metal nanohybrids}

\subsection{Graphene-AuNPs nanohybrids}

An electrochemical immunosensor for carcinoembryonic antigen (CEA) using nanosilver-coated magnetic beads and gold- graphene nanolabels was developed by Chen et al. (2012). The redox-active magnetic nanostructures, based on the combination of a magnetic nanocore, a layer of electroactive poly( $o$-phenylenediamine) (PPD), and a silver metallic shell, displayed good adsorption properties for the attachment of capture antibodies and were integrated into a magnetic carbon paste electrode (CPE). Moreover, these magnetic nanocomposites can efficiently encapsulate the redox electroactive species avoiding the contamination of the electron mediators in the detection solution. AuNP - graphene oxide (GO) conjugated with peroxidase (HRP)labeled detector antibodies were also used as labels in this sandwich-type immunoassay protocol. The doped PPD acted as crosslinkage and mediator for the electron transfer of HRP reduction of $\mathrm{H}_{2} \mathrm{O}_{2}$ monitored by differential pulse voltammetry (DPV). This nanoparticle-based immunocomposites allowed the detection of CEA at a concentration as low as $1.0 \mathrm{pg} \mathrm{mL}^{-1}$ and was successfully applied for its detection in clinical serum specimens.

A disposable electrochemical immunosensor for simultaneous assay of a panel of breast cancer tumor markers was constructed (Ge et al., 2012) using an AuNPs-Gr-modified carbon electrode 
array and alkaline phosphatase (ALP)-labeled detector antibody $\left(A b_{2}\right)$ functionalized $\mathrm{Au}$ cluster (AuCs)-Gr as nanocarriers. The AgNPs deposition, catalyzed by both ALP and AuCs - Gr after the hydrolysis of 3-indoxyl phosphate, was measured by anodic linear sweep voltammetric stripping analysis. This ultrasensitive cancer antigen (CA) multianalyte immunosensor, with limits of detection (LODs) of $1.5 \times 10^{-3} \mathrm{U} \mathrm{mL}^{-1}$ (CA153), $3.4 \times 10^{-4} \mathrm{U} \mathrm{mL}^{-1}$ (CA125), and $1.2 \times 10^{-3} \mathrm{ng} \mathrm{mL}^{-1}$ (CEA), allowed a simple detection method for fast measurement of various tumor markers and avoided crosstalk, which represented significant clinical value for application in cancer screening, providing great potential for convenient pointof-care testing and commercial application.

An electrochemical immunosensor for CEA was developed by immobilizing an HRP-labeled anti-CEA antibody on an AuNPs-Gr composite modified glassy carbon electrode (GCE) (Zhu et al., 2013). HRP was casted on the modified electrode surface not only as a blocker for non-specific sites but also as a catalyzer for redox reaction of hydroquinone (HQ) and $\mathrm{H}_{2} \mathrm{O}_{2}$. The biospecific interaction between CEA and the antibodies assembled on electrode surface resulted in the notably decreased cathodic peak current response of HQ due to the enhanced steric hindrance. Under the optimized conditions, the peak current change derived from the HQ DPV measurements was proportional to the CEA concentration from 0.10 to $80 \mathrm{ng} \mathrm{mL}^{-1}$ with a LOD of $0.04 \mathrm{ng} \mathrm{mL}^{-1}$. In addition, this new immunosensing platform was applied to the determination of clinical serum specimens, with results in agreement with those derived from an enzyme linked immunosorbent assay
(ELISA).

Dharuman et al. (2013) prepared a new electro-reduced graphene and AuNPs (ErGr-AuNP) composite on and indium tin oxide (ITO) electrode to develop a label-free immunosensor for estradiol. Coherent interactions between the ITO, ErGr and AuNPs are responsible for the discrete formation of vertical oriented hetero structures of ErGr-AuNP composite on the ITO which allows a near vertical immobilization of the capture antibody and a low LOD of $0.1 \mathrm{fmol}$ without using any signal amplifiers.

An immunosensor for detecting toxoplasma gondii-specific IgM $(\mathrm{Tg}-\mathrm{IgM})$ was constructed on $\left(\mathrm{Au}-\mathrm{Fe}_{3} \mathrm{O}_{4}\right)$ NPs labeled with detector antibodies as trace labels and AuNPs attached to thionine (Thi) electropolymerized on a nafion - graphene sheets modified electrode (AuNPs - Thi - Nf-GS) as platform for immobilization of toxoplasma gondii antigen ( $\mathrm{Tg}-\mathrm{Ag}$ ) (Fig. 1A) (Jiang et al., 2013). Electrochemical detection was carried out by cyclic voltammetry (CV) in the presence of $\mathrm{H}_{2} \mathrm{O}_{2}$ as HRP substrate (Fig. 1B). The immunosensor exhibited high selectivity, long-term stability, quick response, sensitive detection (LOD, $0.016 \mathrm{AU} \mathrm{mL}^{-1}$ ) and was used to detect $\mathrm{Tg}-\mathrm{IgM}$ in serum samples.

A label-free immunosensor for the simultaneous determination of CEA and $\alpha$-fetoprotein (AFP) was developed by coating reduced GO (rGO)-Thi-AuNPs and rGO-Prussian Blue (PB)-AuNPs nanocomposites, respectively, on an ITO electrode (Jia et al., 2014). The determination was based on the decrease in the response curves of Thi and PB with the concentration of the corresponding antigens due to the formation of the antibody-antigen complex.
B)

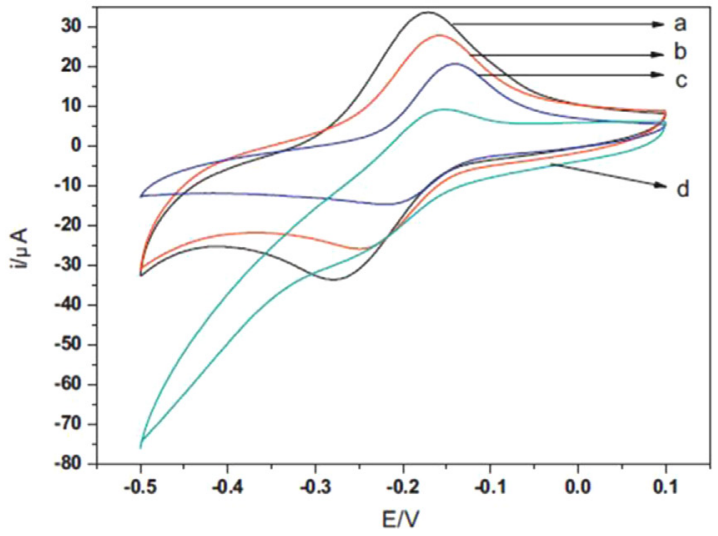

Fig. 1. (A) Schematic representation of a $\mathrm{Tg}-\mathrm{IgM}$ immunosensor based on $\left(\mathrm{Au}-\mathrm{Fe}_{3} \mathrm{O}_{4}\right) \mathrm{NPs}$ and $\mathrm{AuNPs} / \mathrm{Thi} / \mathrm{Nf}-\mathrm{GS}$ nanocomposites. (B) CVs of the modified electrode (a) after incubation with antigen (b) and trace label (c) in the absence (d) and presence of $\mathrm{H}_{2} \mathrm{O}_{2}$. (Reprinted and adapted from Jiang et al. (2013) with permission. Copyright 2013 Elsevier). 
This multiplexed immunosensor enabled the simultaneous determination of both biomarkers with LODs of 0.650 and $0.885 \mathrm{pg} \mathrm{mL}^{-1}$ for CEA and AFP, respectively. The proposed immunosensor avoided labeling either antigens or antibodies, simplified the operation and prevented the cross-talk between the different analytes. Moreover, the methodology was applied to the analysis of clinical serum samples with results correlating well with those provided by ELISA.

A sandwich procalcitonin immunosensor was developed using an rGO-Au nanocomposite film platform and single-walled carbon nanohorns hollow Pt chains - HRP - Thi complexes as signal tags (Liu et al., 2014). The electrochemical response of the immunosensor was monitored by DPV using $\mathrm{H}_{2} \mathrm{O}_{2}$ as enzyme substrate. The LOD of the immunosensor was $0.43 \mathrm{pg} \mathrm{mL}^{-1}$ and it was successfully applied to the determination of procalcitonin in serum samples.

Liu et al. developed an AuNPs-ionic liquid (IL) functionalized rGO immunosensing platform (IL-rGO-AuNPs) for simultaneous electrochemical detection of CEA and AFP (Liu and Ma, 2014). A sandwich-type electrochemical immunosensor was designed based on this IL-rGO - AuNPs nanocomposite platform and using chitosan (CS) coated PB nanoparticles (PBNPs) or cadmium hexacyanoferrate nanoparticles (CdNPs) loaded with AuNPs as distinguishable signal tags. The electrochemical signals were simultaneously measured by DPV at different peak potentials because of the presence of PBNPs and CdNPs. The immunosensor exhibited LODs of $0.01 \mathrm{ng} \mathrm{mL}^{-1}$ for CEA and $0.006 \mathrm{ng} \mathrm{mL}^{-1}$ for AFP, respectively. In addition, results provided in clinical serum samples were consistent with those obtained by an ELISA method, indicating that this immunosensing platform provides a possible application for the simultaneous determination of CEA and AFP in clinical diagnostics. However, the sensitivity achieved with this approach is much lower than that obtained by the same research group when using AuNPs-Thi-rGO and AuNPs-PB-rGO (Jia et al., 2014).

Wang et al. fabricated also an interleukin-6 (IL-6) electrochemical immunosensor using an AuNPs-Gr-silica sol-gel as immobilization biointerface onto an ITO electrode and AuNPsPDA-modified CNTs bioconjugated with HRP and detector antibodies as labels (Wang et al., 2014). This immunosensor, which exhibited a low LOD of $0.3 \mathrm{pg} \mathrm{mL}^{-1}$, provided results in serum samples in agreement with the ELISA method.

Samanman et al. (2015) developed a highly-sensitive label-free electrochemical CEA immunosensor by preparing a cryogel from an AuNPs-graphene-chitosan nanocomposite (AuNPs-Gr-CS) modified on a silver deposited Au electrode. This nanocomposite combined the advantages of graphene, AuNPs (excellent biocompatibility and large surface area) and chitosan (good adhesion, non-toxicity and abundant reactive amino groups) together with the ease to prepare a cryogel (better electron transfer and larger surface area) coupled to a silver deposition, to act as a redox mediator, on an Au electrode. Under the optimal conditions, the decrease in the oxidation peak current measured by CV allowed an extremely low LOD of $2.0 \times 10^{-7} \mathrm{ng} \mathrm{mL}^{-1}$ to be achieved. Moreover, this AuNPs-Gr-CS cryogel electrode, which gave a 1.7 times higher sensitivity and 25 times lower LOD than the non-cryogel electrode, was successfully applied to the analysis of clinical serum samples.

An ultrasensitive and simple immunosensor for the simultaneous detection of four biomarkers was presented by Zhu et al. (2015). This sandwich immunosensor was based on the use of a Gr-AuNPs hybrid film codeposited in one-step onto a GCE as scaffold to immobilize the capture antibody. Hybridization chain reaction (HCR) and biotin/streptavidin, combining with magnetic AuNPs were applied to increase the immobilization amount of signal tags in detection antibody bioconjugates (Fig. 2A). Biotin labeled $\mathrm{Ab}_{2}$ (bio- $A \mathrm{~b}_{2}$ ) was immobilized by gold magnetic nanomaterials $\left(\mathrm{Au}-\mathrm{SiO}_{2}-\mathrm{Fe}_{3} \mathrm{O}_{4}\right)$, then using streptavidin as "bridge" to connect biotin- $A b_{2}$ and biotin-DNA probe 1 , next, DNA probe 2 and bio-DNA probe 3 were added to trigger HCR so as to form long dsDNAs containing abundant biotins and reacted with redox probes labeled streptavidin. The separate peaks corresponding to the different redox probes used: anthraquinone 2-carboxylic acid (AQ), ferrocenecarboxylic acid, Thi and Tris(2,2'-bipyridine-4,4'-dicarboxylic acid)cobalt(III) $\left(\mathrm{Co}(\mathrm{bpy})_{3}{ }^{3+}\right)$ (Fig. 2B), allowed the simultaneous detection of AFP, CEA, CA125 and prostatic specific antigen (PSA) in single-pass DPV scan with LODs of 62, 48, 77 and $60 \mathrm{fg} \mathrm{mL}^{-1}$, respectively.

An impedimetric immunosensor for C-reactive protein (CRP) was fabricated by functionalizing an ITO microdisk electrode array chip with rGO - AuNPs hybrid (Yagati et al., 2016). The impedimetric measurements obtained in the presence of $\left[\mathrm{Fe}(\mathrm{CN})_{6}\right]^{3-14-}$

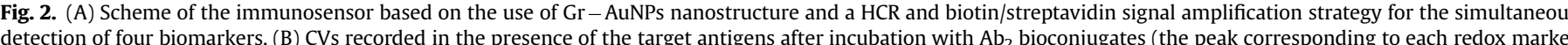
is identified). (Reprinted and adapted from Zhu et al. (2015) with permission. Copyright 2015 Elsevier). 
were associated with LODs of 0.06 and $0.08 \mathrm{ng} \mathrm{mL}^{-1}$ in PBS and human serum, respectively.

Very recently, Zhang et al. (2015a) developed a double signal electrochemical human IgG immunosensor based on AuNPs - PDA - rGO as a sensor platform and AgNPs/carbon nanocomposite $(\mathrm{Ag} / \mathrm{CNC})$ as signal probe and catalytic substrate. In this sandwich immunosensor, the $\mathrm{Ag} / \mathrm{CNC}$ functionalized bioconjugates were captured on HIgG - $\mathrm{Ab}_{1} / \mathrm{AuNPs}$ - PDA - rGO surface and the electrochemical double-signal strategy was employed, one electrochemical signal directly originated from oxidation of $\mathrm{Ag}-\mathrm{CNC}$, and the other coming indirectly from reduction of benzoquinone which was produced from the reaction of $\mathrm{H}_{2} \mathrm{O}_{2}$ and $\mathrm{HQ}$ by catalysis of $\mathrm{Ag}-\mathrm{CNC}$. Under the optimized conditions, the DPV responses allowed a LOD of $0.001 \mathrm{ng} \mathrm{mL}^{-1}$ in the presence of both analytes, and the immunosensor was successfully applied in the analysis of human IgG in real samples.

A label-free electrochemical immunosensor for neuron-specific enolase based on the electrochemical catalysis of nickel hexacyanoferrates nanoparticles (NiHCFNPs) (in the presence of dopamine (DA)), which were assembled on porous gold nanocrystals (AuNCs) modified GCE and further coated by AuNPs-GSs was described by Han et al. (2012). The detection is based on the change of the anodic peak current of DA obtained by CV before and after the antigen-antibody reaction, due to the immunocomplex hindering the access of the redox probe to the electrode. Under optimal conditions, the electrochemical immunosensor exhibited a LOD of $0.3 \mathrm{pg} \mathrm{mL}^{-1}$. The great sensitivity of this immunosensor relies on the multiple signal amplification involved: dual-effects of $\mathrm{Au}-\mathrm{GS}$ for enhancing the electroactivity of NiHCFNPs layer and increasing the amount of capture antibody loading, and the excellent catalytic activity of NiHCFNPs towards DA.

A competitive electrochemical immunosensor for the detection of human IL-6 was developed based on ErGO and an AuPdNPs immunosensing platform onto a heated CPE (HCPE) and AgNPs functionalized polystyrene bionanolabels (Lou et al., 2014). The silver component acting as the detector target was quantified by linear sweep voltammetry (LSV) at an electrically HCPE to further improve the sensitivity. The immunosensor exhibited a LOD of $0.059 \mathrm{pg} \mathrm{mL}^{-1}$. The proposed method showed good precision, broad linear range, acceptable stability and high reproducibility, and could be used for the detection of IL- 6 in real samples.

A sandwich immunosensor for CEA was developed using GS both for the immobilization of $A b_{1}$ and as a tracer to label the $A b_{2}$. The $A b_{1}$ was immobilized on AuNPs-GS and $A g-A u N P s$ assembled on graphene conjugated with the $\mathrm{Ab}_{2}$ were used as molecular tags. In both cases 1,5-diaminonaphthalene, adsorbed onto GS through $\pi-\pi$ stacking, was used to prepare the hybrid nanomaterials. The peak obtained at $0.46 \mathrm{~V}$ (vs. SCE) after CEA recognition attributed to the oxidation of $\mathrm{Ag}$ to $\mathrm{Ag}_{2} \mathrm{O}$ from the coupled NPs was used as electrochemical signal (Huang et al., 2015). This enzyme-free immunosensor displayed a low LOD $\left(8 \mathrm{pg} \mathrm{mL}^{-1}\right)$ and was successfully used in determining CEA in spiked human serum.

\subsection{Graphene-other metallic NPs nanohybrids}

Cai et al. (2012) developed an electrochemical immunosensor for CEA using a signal amplification strategy with a nanotubular mesoporous $\mathrm{PdCu}$ alloy $(\mathrm{NM}-\mathrm{PdCu})$. The $\mathrm{NM}-\mathrm{PdCu}$ provided a large surface area to immobilize biomolecules spatially patterned and enhanced the immobilization capability of CEA, which ensured the biomolecules would adopt a more flexible orientation. This immunosensor was fabricated through strong binding of the capture antibody on $\mathrm{CS}-\mathrm{PdCu}$ immobilized electrostatically on GCEs functionalized previously with a mixture of sulfonated graphene sheets and Thi. Since the antigen-antibody complex coating on the surface of the electrode inhibited the electron-transfer, the decrease in the Thi reduction peak measured by CV in the presence of CEA was taken as analytical signal. The immunosensor exhibited a low LOD of $4.86 \mathrm{pg} \mathrm{mL}^{-1}$ and satisfactory results in real serum samples analysis.

Yu et al. (2013) fabricated a sensitive label-free immunosensor for kanamycin detection using Ag hybridized mesoporous $\mathrm{Fe}_{3} \mathrm{O}_{4}$ NPs (Ag@ $\mathrm{Fe}_{3} \mathrm{O}_{4}$ NPs) and Thi mixed GS (Thi-GS)-modified GCE to enhance the electrochemical response. This label-free immunosensor measured the SWV Thi peak current change before and after the antigen-antibody reaction. The immunosensor showed a low LOD (15 $\left.\mathrm{pg} \mathrm{mL}^{-1}\right)$ and satisfactory evaluation for pork meat sample analysis.

A high throughput immunosensor based on multi-label strategy and a novel array electrode was developed for simultaneous detection of six biomarkers for hepatocellular carcinoma (Yang et al., 2014a). Graphene nanosheet-PtPd bimetallic nanocomposites (PtPdNPs - GS) labeled with HRP and the detector antibodies were used as bimolecular carriers not only to increase the antibody loading, but also to amplify the signal by using the PtPdNPs catalytic activity. As shown in Fig. 3, the designed array electrode, composed of three detection spots and a control one, could detect simultaneously the six biomarkers. Two different capture antibodies were immobilized on each detection spot and the corresponding detector antibodies were labeled with the redox probes Thi and $A Q$ respectively. Based on the sandwiched immunoreactions, each individual protein could be detected with the distinct voltammetric peaks, whose position and size reflected the identity and level of the corresponding antigen.

A sandwich electrochemical immunosensor was also reported for the detection of secretoneurin based on $\mathrm{Pb}^{2+}$-functionalized rGO-tetraethylenepentamine (rGO-TEPA) conjugated with detector antibodies as nanocarriers and a GCE modified with AuNPs - GS as electrochemical platforms for the immobilization of the capture antibody (Yuan et al., 2015). The immunoreactions were followed by detecting by DPV the metal ions in the bioconjugates without acid dissolution or preconcentration steps required by stripping voltammetry. This immunosensor exhibited an ultralow LOD of $0.33 \mathrm{pg} \mathrm{mL}^{-1}$ and applicability in clinical serum samples.

\section{Graphene-metal oxide nanohybrids}

Graphene's high specific area $\left(2630 \mathrm{~m}^{2} \mathrm{~g}^{-1}\right)$ together with its unique two-dimensional crystal structure makes it extremely useful as support material for metal oxide nanoparticles in the development of electrochemical biosensors. In these sensors, metal oxide nanoparticles act as the active site to enhance specificity and sensitivity while graphene offers fast electron transfer in the electrochemical reaction. Metal oxide nanostructures can be grown on graphene sheets through different procedures including in situ chemical synthesis, hydrothermal processes, microwave heating, and electrodeposition. The growth mechanism is based on the attraction of positively-charged metal/metal oxide ions by the polarized bands of the functional groups on graphene (such as $\mathrm{OH}$, carboxylic $\mathrm{C}=\mathrm{O}$, carboxylate $\mathrm{O}=\mathrm{C}=\mathrm{O}, \mathrm{C}-\mathrm{O}$, and $\mathrm{O}-\mathrm{C}-\mathrm{O}$ ). The main characteristics of the described immunosensing platforms based on graphene/metal oxides nanostructures are summarized in Table 1.

4.1. Graphene-metal oxide nanoparticles hybrids as electrode surface modifiers in electrochemical immunosensors

An interesting example on the modification of electrode sur- Q431 faces with metal oxides-graphene nanocomposites is that 


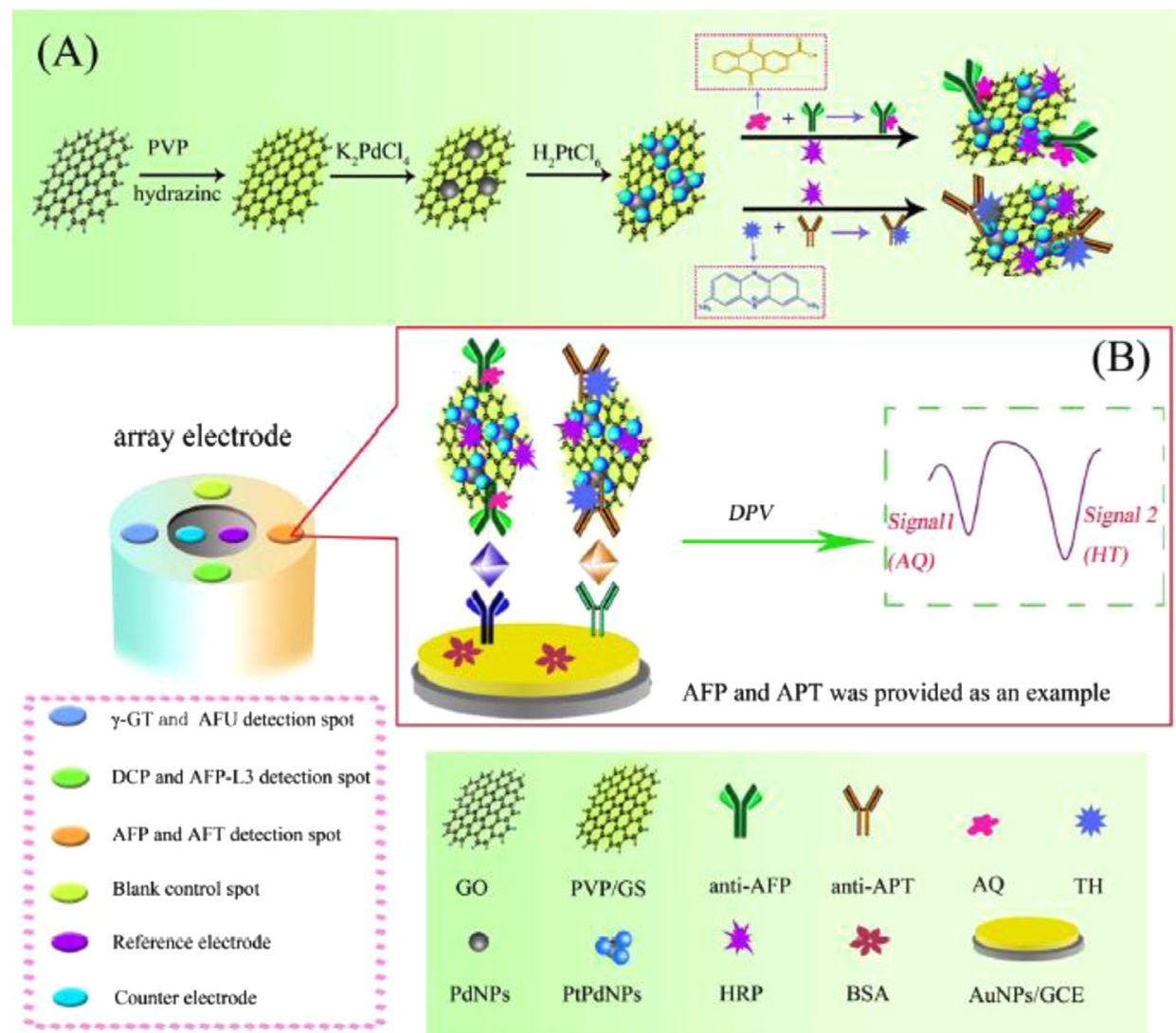

Fig. 3. Preparation procedure of HRP and PtPd -GS labeled redox probe branched antibodies (A). Schematic illustration of the stepwise immunosensor fabrication process and interaction of antibody-antigen (B). (Reprinted from Yang et al. (2014a,2014b) with permission. Copyright 2014 NPG).

Table 1

Electrochemical immunosensors for clinical biomarkers based on graphene-metal oxide nanohybrids.

\begin{tabular}{|c|c|c|c|c|c|c|}
\hline Graphene-metal oxide hybrid & Analyte & LOD & RSD (\%) & Preparation time ${ }^{*}$ & Lifetime & Reference \\
\hline $\mathrm{Ab}_{1} / \mathrm{Fe}_{3} \mathrm{O}_{4}-\mathrm{GO} / \mathrm{ITO}$ & CEA, AFP & $1 \mathrm{pg} / \mathrm{mL}$ & $\begin{array}{l}9.8(\mathrm{AFP}) \text { and } 7.2(\mathrm{CEA}) \\
(n=6)\end{array}$ & $\sim 30 \mathrm{~h}$ & 17 days & Tang et al. (2011) \\
\hline $\mathrm{Ab}_{1} / \mathrm{ZnO}-\mathrm{GO} / \mu \mathrm{PAD}$ & hCG, PSA, CEA & $\begin{array}{l}0.0007 \mathrm{mIU} / \mathrm{mg}, 0.35 \mathrm{pg} / \mathrm{mL} \\
\text { and } 0.33 \mathrm{pg} / \mathrm{mL} \text {, respectively }\end{array}$ & $\begin{array}{l}5.5,5.7 \text { and } 5.3 \text {, respec- } \\
\text { tively }(n=7)\end{array}$ & $\sim 16 \mathrm{~h}$ & 30 days & Sun et al. (2015) \\
\hline $\mathrm{Ab}_{1} / \mathrm{AgNPs}-\mathrm{SiO}_{2}-\mathrm{GO} / \mathrm{GCE}$ & Ethinylestradiol & $65 \mathrm{pg} / \mathrm{mL}$ & $4.5(n=10)$ & 11 days & 15 days & $\begin{array}{l}\text { Cincotto et al. } \\
\text { (2016) }\end{array}$ \\
\hline $\begin{array}{l}\mathrm{Fe}_{3} \mathrm{O}_{4}-\mathrm{GrQDs} / \mathrm{Ab}_{2} \\
-\mathrm{Cu}-\text { apoferritin }\end{array}$ & $\begin{array}{l}\text { Avian leukosis virus sub- } \\
\text { group J }\end{array}$ & $115 \mathrm{TCID}_{50}$ & $2.18(n=6)$ & 4 days & 30 days & $\begin{array}{l}\text { Wang et al. } \\
\text { (2013) }\end{array}$ \\
\hline $\mathrm{Ab}_{2} / \mathrm{MSN} / \mathrm{GO}$ & CA153 & $2.8 \times 10^{-4} \mathrm{U} / \mathrm{mL}$ & $4.1(n=5)$ & 8 days & 1 month & Ge et al. (2014) \\
\hline
\end{tabular}

* Includes nanomaterial preparation and electrode modification. CA, cancer antigen; CEA, carcinoembryonic antigen; AFP, $\alpha$-fetoprotein GCE, glassy carbon electrode; GO,

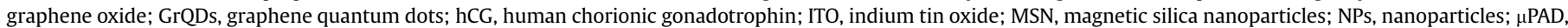

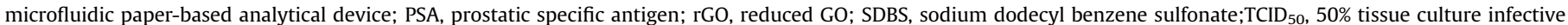
dose.

described by Tang et al. (2011) consisting on a magneto-controlled graphene immunosensor platform for the simultaneous multiplexed electrochemical determination of the tumor markers CEA and AFP in biological fluids using a flow through system. Primary anti-CEA and anti-AFP antibodies were immobilized on $\mathrm{Fe}_{3} \mathrm{O}_{4}$ nanoparticle-coated graphene nanosheets, and multifunctional nanogold hollow nanospheres were used as distinguishable signal tags (Fig. 4). In this way, magnetic graphene nanosheets were used as substrate for the immobilization of the biomolecules, and as means for the rapid separation and purification system. Thus, by using a magnetic field gradient, antibodies bound to magnetic NPs can be pulled from one laminar flow path to another and selectively removed from biological fluids without washing steps. This approach allowed much lower LODs (Table 1) than that developed by Liu and Ma (2014) using an IL-rGOAu support matrix, while its sensitivity is similar to that obtained by Jia et al. (2014) using AuNPs - Thi - rGO and AuNPs - PB - rGO as support matrixes on ITO electrodes (see Section 3.1).

More recently, a multiplexed enzyme-free electrochemical immunoassay was constructed on a microfluidic paper-based analytical device resulting in a low cost, easy to use, low reagent and sample consumption, portable and disposable system which was applied to the multiplexed determination of three tumor markers (Sun et al., 2015). ZnO nanorods-modified, rGO-paper electrodes were used as sensor platforms and composites of rGO and BSA-stabilized silver nanoparticles (Ag@BSA) as signal labels. High conductivity rGO at the electrode surface improved the electron transfer rate, biocompatible $\mathrm{ZnO}$ nanorods large surface area provided abundant sites for capture probes binding, and the current signal was generated from the reduction of $\mathrm{H}_{2} \mathrm{O}_{2}$ further 


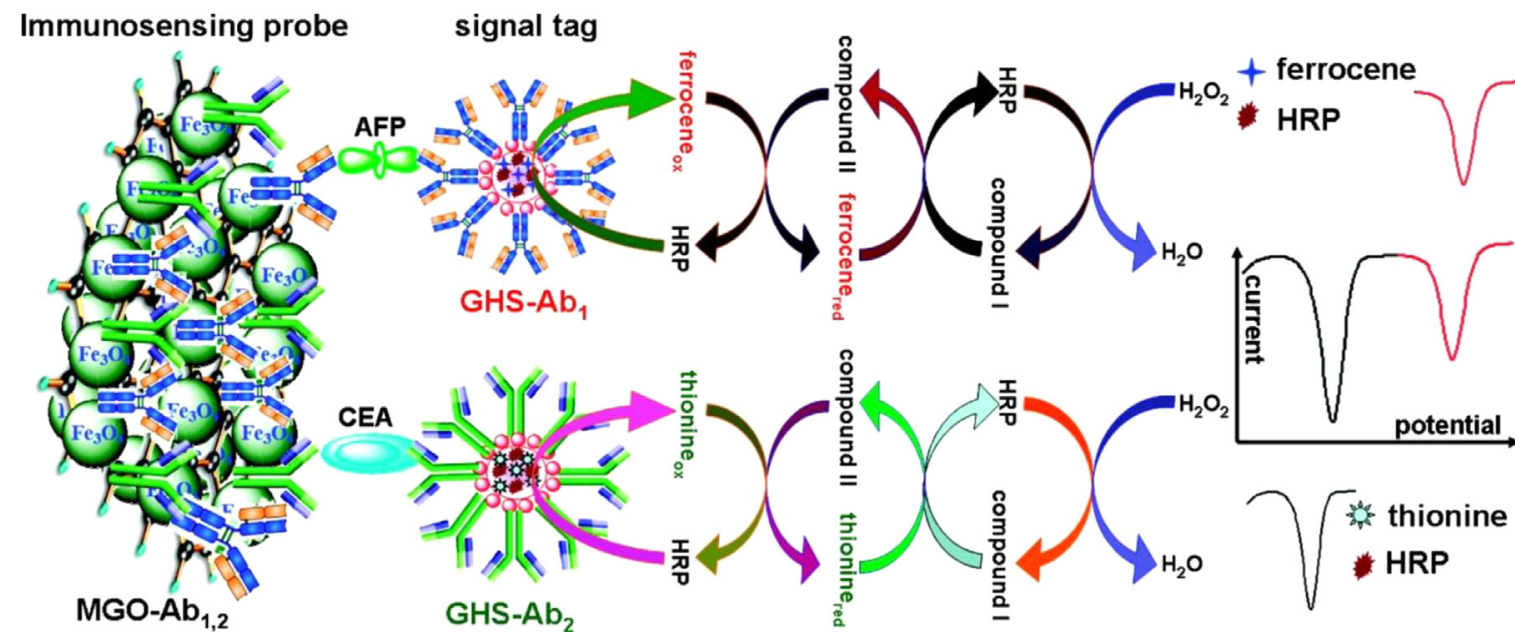

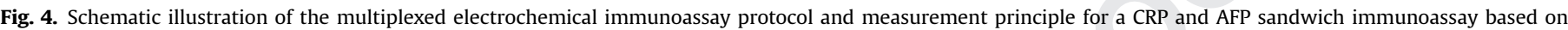
$\mathrm{Fe}_{3} \mathrm{O}_{4}-\mathrm{GO}$ modified ITO electrodes. (Reprinted from Tang et al. (2011) with permission. Copyright 2011 ACS Publications).

amplified by a subsequent signal labels-promoted deposition of silver accelerating electron transfer and catalyzing the reduction of $\mathrm{H}_{2} \mathrm{O}_{2}$. On the other hand, a competitive immunosensor for the determination of the hormone ethinylestradiol (EE2) in spiked urine samples has been described (Cincotto et al., 2016) using a GCE modified with a AgNPs - $\mathrm{SiO}_{2}-\mathrm{GO}$ hybrid activated by grafting a diazonium salt of 4-aminobenzoic acid for the covalent binding of antiEE2 to the activated carboxyl groups. Taking into account the high time needed to prepare the hybrid modifier (around 8 days), it would be interesting to know its stability with time and under which conditions it should be preserved (data almost never given in any paper).

4.2. Graphene-metal oxide nanoparticles hybrids as labels in electrochemical immunosensors

Graphene-metal oxide nanohybrids have scarcely been used as labels in electrochemical immunosensors. Graphene QDs
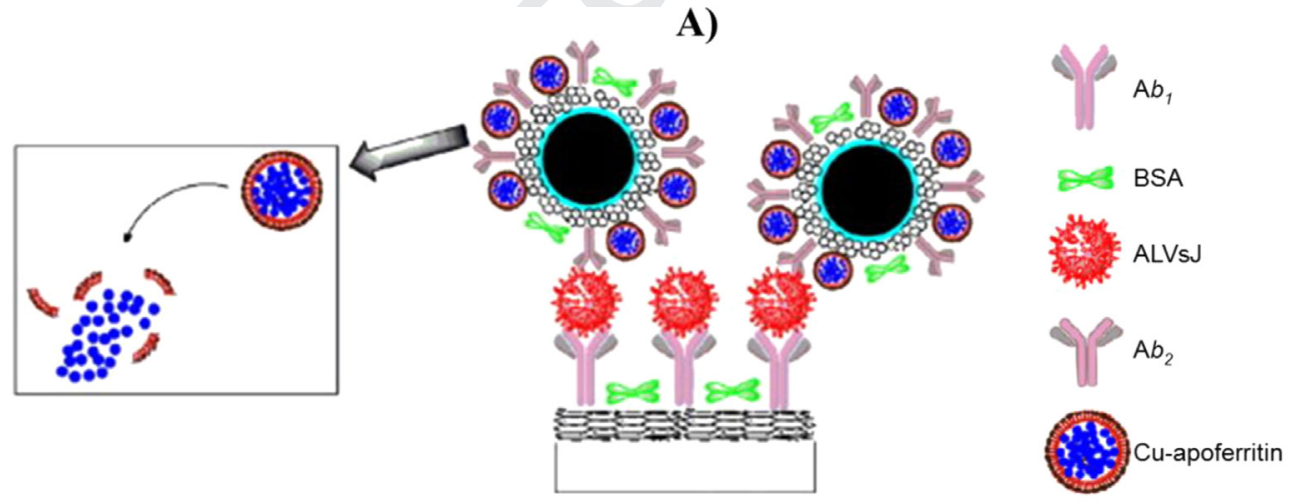

B)
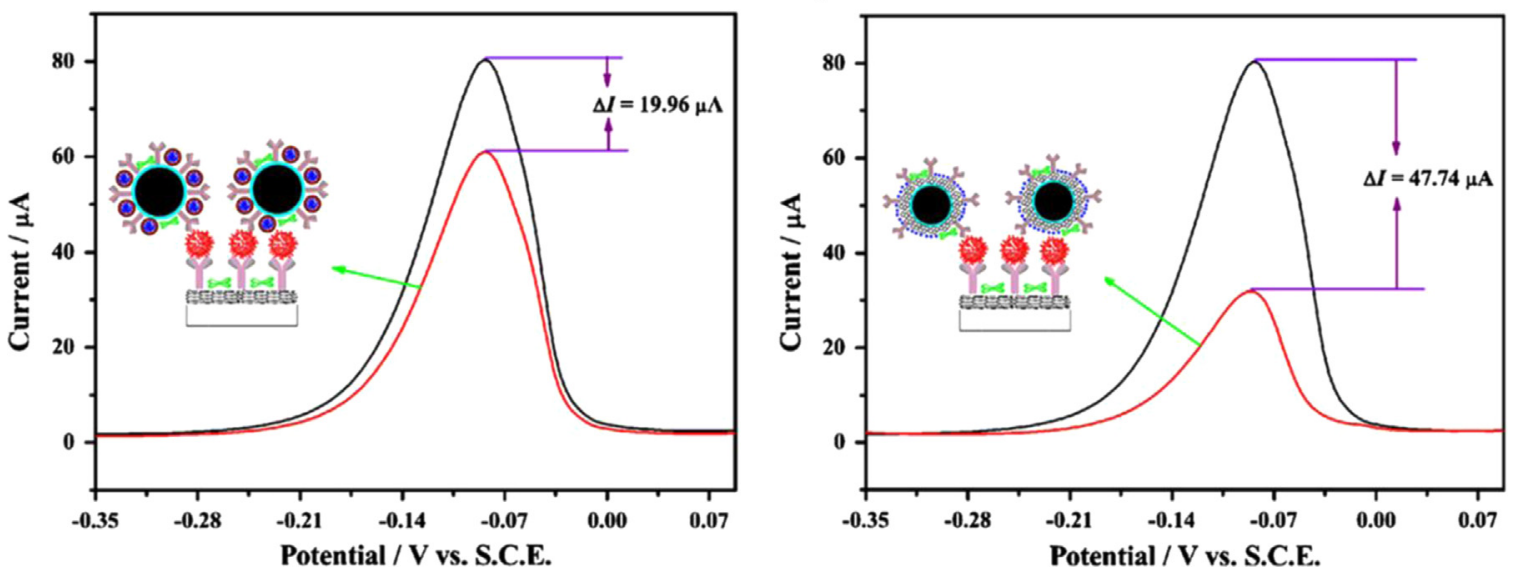

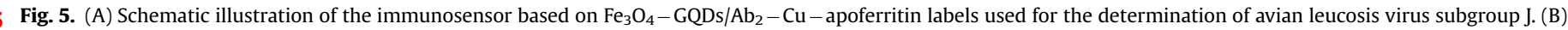

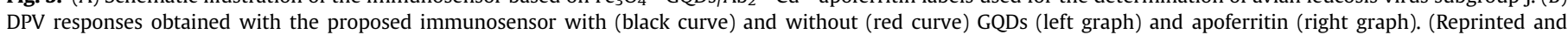

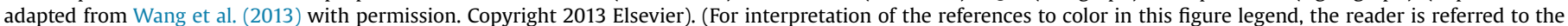
web version of this article.) 
compounded with $\mathrm{Fe}_{3} \mathrm{O}_{4}$ and apoferritin-encapsulated $\mathrm{Cu}$ nanoparticles were used for signal amplification in the development of a sandwich electrochemical immunoassay for the detection of avian leucosis virus subgroup J (ALVs-J) (Wang et al., 2013). As can be seen in Fig. 5A, graphene QDs were also used for the immobilization of the primary ALVs - J antibodies. After the sandwich-type assembly, $\mathrm{Cu}$ was released from the apoferritin cavity and detected by DPV on a Bi-coated GCE electrode (Fig. 5B). Graphene QDs allowed a great number of primary antibodies to be loaded onto the immunosensor, while the introduction of $\mathrm{Cu}-$ apoferritin NPs increased the loading amount of electroactive probes, both factors contributing to the amplification of the electrochemical signal.

\section{Graphene-CNTs nanohybrids}

CNTs also constitute interesting nanomaterials for the preparation of electrochemical sensors with improved electron transfer at the electrode surface, increased electrode surface area to load larger amounts of biomolecules and enhanced sensitivities. Table 2 summarizes the main characteristics of the electrochemical immunosensors developed using CNTs-Gr nanocomposite hybrids for clinical biomarkers.

Liu et al. (2012) fabricated a MWCNT - Gr composite electrode interface by using a layer-by-layer assembly. This methodology relied on the electrostatic adsorption between positively charged poly(diallyldimethylammonium chloride) and negatively charged MWCNTs and Gr. The result was a three-dimensional uniform and highly porous MWCNT-Gr composite network electrode interface. It was demonstrated that the composite film improved the interfacial electron transfer when compared with that of the $\mathrm{Gr}$ or the MWCNTs-modified GCE. The hybrid was applied to the determination of human IgG in human serum samples in a sandwich configuration using HRP-conjugated IgG as the detection antibody and $\mathrm{HQ}$ as the electron mediator with good results in terms of selectivity, stability, reproducibility and accuracy.

The satisfactory properties of these nanostructures were improved by decorating the MWCNTs-Gr composites with AuNPs. Lu et al. (2012) described an immunosensor for the detection of human chorionic gonadotrophin (hCG) where AuNPsdotted MWCNTs - Gr composites were immobilized on a working GCE seeking an increase in the surface area to capture a large amount of primary antibodies and improve the electron transfer rate. They also used functionalized mesoporous nanoparticles as bionanolabels which were composed of mesoporous silica nanoparticles coated with AuNPs through thionine linking where the HRP-labeled secondary antibody was well-adsorbed. Again, an immunosensor with good reproducibility and stability was obtained and applied to the determination of the target analyte in human serum samples. Feng et al. (2013) described a label-free electrochemical immunosensor for the detection of CEA using a GCE modified with a multilayer film composite made of $\mathrm{PB}, \mathrm{Gr}$ and CNTs by electrodeposition and assembly techniques. Again, AuNPs were electrostatically absorbed on the surface of the film and used in the capture antibody immobilization, while PB acted as signaling molecule. The formation of the antibody-antigen complexes partially inhibited the electron transfer and decreased the PB peak current. The multi-layer film of (PB-AuNPs-rGO-MWCNTs) was said to show higher stability and to provide higher amount of immobilized capture antibody also with rGO - MWCNTs improving the electron transfer ability of PB. Fang et al. (2014) increased the electron transfer rate and improved the surface area to capture a large number of primary antibodies by layer-by-layer coating $\mathrm{Gr}$, MWCNTs, CS and glutaraldehyde (GA) on a GCE to detect procalcitonin, a marker for the identification of severe bacterial infections. They prepared the trace tag by loading HRP-labeled secondary antibody with AuNPs coated with mesoporous silica nanoparticles through thionine linking. The immobilized chitosan on the MWCNTs - Gr nanocomposite modified electrode was said to form a uniform film with unique physical and chemical features that could increase the surface loading amount of the capture antibody through GA with a described as excellent electrochemical response and selectivity to the antigen. The proposed method was demonstrated to show a better accuracy, stability, reproducibility and sensitivity than other techniques.

The cardiac marker Troponin I (cTnI) has been label-free detected by using a one-step microwave-assisted unscrolling of CNTs to form functionalized rebar graphene (f-RGr) (Tuteja et al., 2014). Rebar graphene is a graphene-nanotube hybrid material containing large, flexible and conductive transparent sheets of graphene that are much easier to manipulate than pure graphene. CNTs act as reinforcing bar (rebar), toughening the Gr through both $\pi-\pi$ stacking domains and covalent binding where the CNTs partially unzip and form seamless 2D conjoined hybrid (Yan et al., 2014). In Tuteja's paper, a novel approach for electromagnetic microwave assisted unscrolling of MWCNTs to form f-RGr is presented, its potential application being demonstrated in label-free immunosensing for the detection of cTnI. Well-characterized f-RGr functionalized with specific anti-cTnI antibodies via available functional carboxylic groups on f-RGr was immobilized on interdigitated electrodes, and then used as a biochip device for the detection of the cardiac marker. In the RGr nanocomposite, the CNTs form networks that serve as bridges across graphene boundaries and provide greater surface area resulting in improved electronic properties as compared to MWCNTs. The developed biochip was demonstrated to offer good selectivity, and high sensitivity in comparison to other conventional methods, which was mainly attributed to the electron mobility of the f-RGr channel with highly specific antibodies. In the f-RGr based FET sensor, RGr acted as the channel where resistance was monitored with respect to voltage in a concentration dependent manner of the target

Table 2

Electrochemical immunosensors for clinical biomarkers based on graphene-CNT nanohybrids.

\begin{tabular}{|c|c|c|c|c|c|c|}
\hline Graphene-CNT hybrid & Analyte & LOD & RSD (\%) & Preparation time* & Lifetime & Reference \\
\hline MWCNT - Gr/GCE & $\operatorname{IgG}$ & $0.2 \mathrm{ng} \mathrm{mL}^{-1}$ & $3.4(n=3)$ & $>9 h$ & 5 weeks & Liu et al. (2012) \\
\hline AuNPs - MWCNT - Gr/GCE & hCG & $0.0026 \mathrm{mIU} \mathrm{mL}^{1}$ & $4.8(n=4)$ & $>32 \mathrm{~h}$ & 30 days & Lu et al. (2012) \\
\hline AuNPs - MWCNT - Gr/GCE & CEA & $60 \mathrm{pg} \mathrm{mL}^{-1}$ & $5.40(n=3)$ & $>36 \mathrm{~h}$ & 3 weeks & Feng et al. (2013) \\
\hline $\mathrm{GA}-\mathrm{CS}-\mathrm{MWCNTS}-\mathrm{GR} / \mathrm{GCE}$ & Procaciltonin & $0.5 \mathrm{pg} \mathrm{mL}^{-1}$ & $6.5(n=5)$ & $>39 \mathrm{~h}$ & 15 days & Fang et al. (2014) \\
\hline $\mathrm{f}-\mathrm{RGr} / \mathrm{AuFET}$ & CTnI & $1 \mathrm{pg} \mathrm{mL}^{-1}$ & - & $>1 \mathrm{~h}$ & - & Tuteja et al. (2014) \\
\hline CS - PTA - PB - MWCNTs - GO/GCE & ST6Gal-I & $3 \mathrm{pg} \mathrm{mL}^{-1}$ & $2.00(n=5)$ & $>3$ days & 2 weeks & Zhang et al. (2015b) \\
\hline AuNPs - CS - MWCNT - Gr/GCE & EBNA-1 & $0.7 \mathrm{pg} \mathrm{mL}^{-1}$ & $4.0(n=5)$ & $>37 \mathrm{~h}$ & 9 days & Song et al. (2014a,2014b) \\
\hline
\end{tabular}

${ }^{*}$ Includes nanomaterial preparation and electrode modification. CEA, carcinoembrionic antigen; CS, chitosan; CTnI, cardiac troponin I; EBNA-1, Epstein Barr virus nuclear

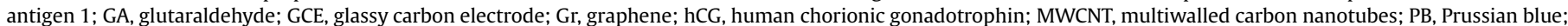
PTA, a derivative of 3, 4, 9, 10-perylenetetracarboxylicdianhydride; ST6Gal-I, $\beta$-galactoside $\alpha$-2, 6 -sialyltransferase. 
antigen cTnI. A change in resistance was observed due to the charges induced in the $\mathrm{f}-\mathrm{RGr}$ channel with the formation of the antibody-antigen immunocomplex, the conductance decreasing gradually with the increase in concentration of highly charged cTnI protein.

Zhang et al. (2015b) described an immunosensor for the determination of $\beta$-galactoside $\alpha-2,6$-sialyltransferase (ST6Gal-I), a regulator of tumor cell survival, in serum samples. This sensor was founded on a PB-based hybrid nanocomposite consisting of GO, MWCNTs, a derivative of $3, \quad 4, \quad 9, \quad 10-$ perylenetetracarboxylicdianhydride called PTA and CS. The abundance of amino groups from CS and PTA favored a dense adsorption of AuNPs on the surface of the nanocomposite thus providing a large available surface area for the immobilization of the capture antibody. PB served as electroactive and biocompatible redox probe. The nanocomposite showed a large surface active area, high catalytic activity, and good stability (Table 2). AuNPs were used to realize the antibody immobilization and enhance the sensitivity of the immunosensor due to their large surface area, strong adsorption ability, high conductivity, and favorable biocompatibility. As
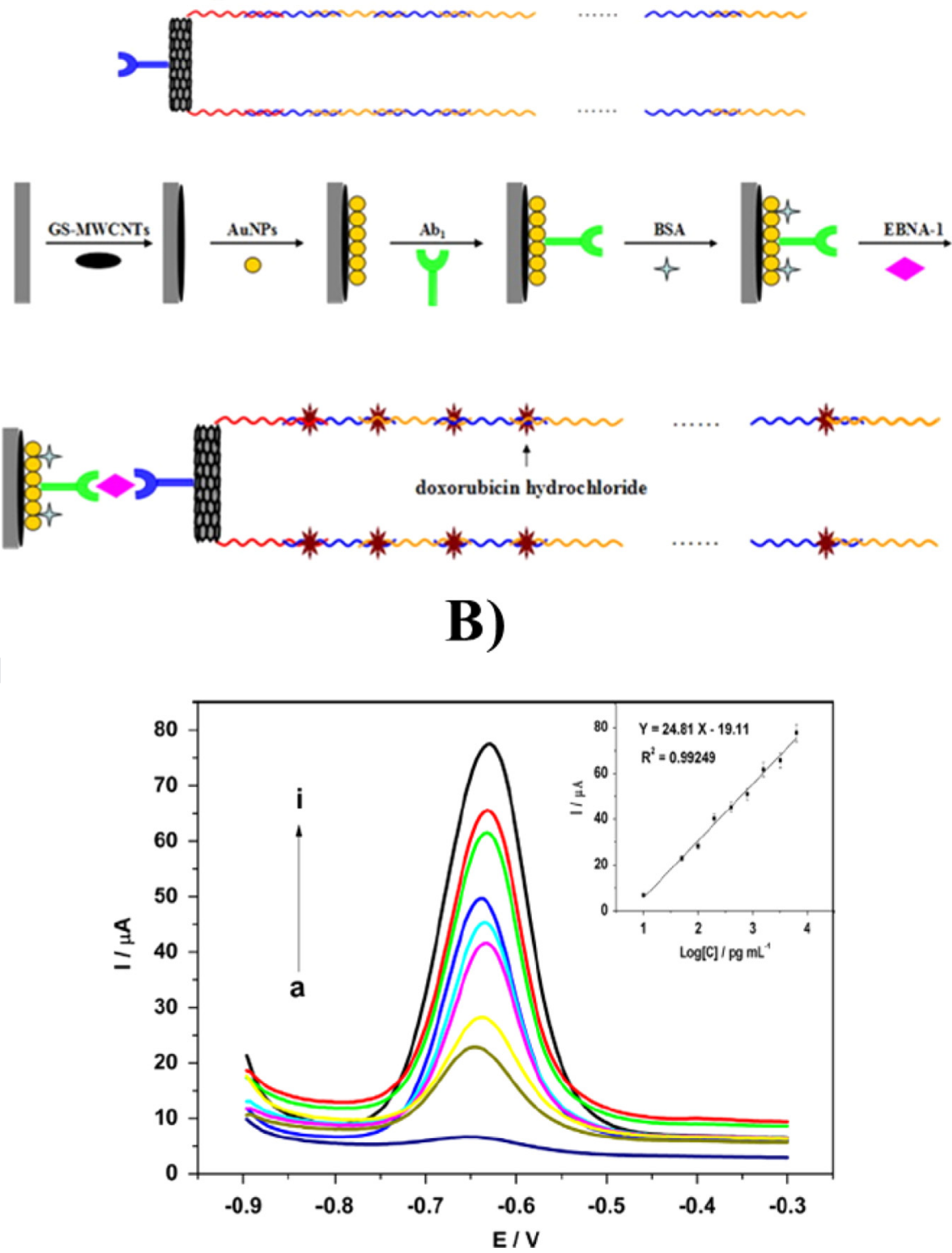

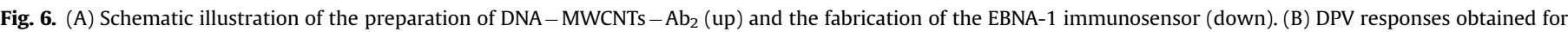
different EBNA-1 concentrations at the developed immunosensor in the concentration range from 0 to $6400 \mathrm{pg} \mathrm{mL}^{-1}$; inset: corresponding calibration plot. (Reprinted and adapted from Song et al. (2014a,2014b) with permission. Copyright 2014 Elsevier). 
occurs with other immunosensors described in this section, this one is said to be an specific and sensitive electrochemical immunosensor that, when compared with the analysis performance of other methods, exhibits higher sensitivity and a wider linear range for the detection of the target analyte with good precision, stability and reproducibility.

A somewhat different approach based on a DNA HCR has been described by Song et al. (2014a) for the determination of the cancer marker Epstein Barr virus nuclear antigen 1 (EBNA-1). Again, a suspension of GSs and MWCNTs was prepared with the aid of CS and used to modify a GCE. AuNPs were then electrodeposited onto the surface of the modified electrode by CV to immobilize the capture antibodies. For the detection step, DNA coated carboxyl MWCNTs-modified secondary antibodies were used. The dsDNA was formed from HCR between initiator strands $\left(S_{0}\right)$ on the $S_{0}-$ MWCNTs $-A_{2}$ and a stable mixture of two alternating hairpins (Fig. 6A). Then, doxorubicin hydrochloride was used as electroactive indicator which intercalated into de CG-GC steps between the HCR products producing an electrochemical signal monitored by DPV (Fig. 6B). As in other examples, MWCNTs - Gr and AuNPs were assembled onto the GCE surface to enhance the conductivity and effective surface area, respectively, which increased the amount of antibodies immobilized on the sensor. The novel amplification strategy used here exhibited a low LOD (Table 2).

\section{GLNs nanohybrids}

Although much less explored than graphene-based, GLNs nanohybrids have demonstrated great potential also as electrode materials in the development of promising non-enzymatic sensors (Yang et al., 2014b; Sun et al., 2014; Xia et al., 2014; Gu et al., 2015) and enzymatic biosensors (Song et al., 2014b). However, only one example of an immunosensor for a clinical biomarker has been found in the literature. It was developed to detect IL-6 using as labels a multifunctional BN nanosheets - Au nanocluster nanocomposite (BN-GNC) fabricated using PDDA as a stabilizer and a linker (Yang et al., 2013). The resulting PDDA-BN-GNC composite was subsequently used for the immobilization of detector antibodies to fabricate PDDA-BN-GNC- $\mathrm{Ab}_{2}$ bioconjugates. The electrochemical signal from the reduction of the electroactive $\mathrm{AuCl}_{4}^{-}$ generated by electrochemical oxidation of nanocrystals in $\mathrm{HCl}$ solution was tested using SWV. The proposed assay showed a low LOD $\left(1.3 \mathrm{pg} \mathrm{mL}^{-1}\right)$.

Among 2-D layered nanomaterials, TMDs have also raised a great interest in the latter years due to their promising properties. In fact, their large surface area and excellent electronic, mechanical and catalytic properties have contributed to increase their popularity in the development of electrochemical sensors. As an example of this interest, a recent review by Su et al. (2015) summarizes recent advances on using graphene and $\mathrm{MoS}_{2}$ in the development of electrochemical sensors for small molecules, proteins, nucleic acids and cells detection, including sections devoted to relevant clinical molecules. However, no examples of immunosensors based on the use of TMDs have been found. As concluded by these authors, hybrid nanostructures based on graphene and $\mathrm{MoS}_{2}$ provide an efficient way for broadening and enhancing the performance of 2D nanomaterials in sensing applications. The challenges to be considered here include the control of the morphology of functional groups together with the definition of accurate and efficient immobilization of biomolecules on the surface of graphene and $\mathrm{MoS}_{2}$, and the improvement of the stability of $\mathrm{MoS}_{2}$ which is easily oxidized during the preparation process.

\section{Conclusions and future prospects}

Recent frontier research on the rational design of functional hybrid 2D-nanomaterials composes mainly of graphene, coupled with electroanalytical methods, has led to great advanced applications, highlighted in this review, demonstrating the promising capabilities of these nanomaterials in electrochemical immunosensing. Therefore, it is expected that 2D-nanohybrids with their multifunctionalities and multimodalities will continue to proliferate in the field of electrochemical immunosensors.

Although electrochemical immunosensors based on 2D-hybrid nanomaterials may offer various advantages for enhancing and superseding the capabilities of current analytical methodologies by permitting rapid and highly accurate analysis, this field is still new and there are many points to be addressed.

First of all, significant efforts should be devoted to develop new methods for facile synthesis of 2D-hybrid nanomaterials with high level of uniformity and reproducibility which lead to maximization of their outstanding properties and their functionalization with defined structure and properties. The size, surface state and wettability of nanomaterials are indeed of great importance. Currently available graphene materials are usually non-uniform in their 2D sizes, which should influence the performances of electrochemical sensors, such as sensitivity, stability and reproducibility. On the other hand, defects produced during the preparation process should largely affect electronic properties of graphene. Moreover, when designing hybrid 2D-nanomaterials great attention must be paid toward optimizing the intrinsic properties of the components simultaneously with the interface structure and mechanisms of electronic coupling, some of which are still unknown.

It is well known that some of the promising properties of the 2D-graphene hybrid nanomaterials are associated with the singlelayer graphene sheets. In this sense, it remains important challenges: fabrication of graphene sheets with high-quality, tailoredfunctionalization, precise and fine-tunable structure and properties in a more cheap, effective and simple way; pay attention to the control distribution, amount, and affinity to graphene sheets, also to the nanohybrids' dispersibility and functionality and through investigation of the mechanisms of probes interacting with graphene or decorated graphene. Exploiting biofunctionalized products with various materials through covalent or non-covalent interactions and to find nanomaterials with sufficient binding sites for biomolecules should be of high interest for the biosensing field. To broaden the scope of their bioapplications in the future and open new probabilities for fundamental research, constructing new hybrid nanocomposites with ideal building blocks such as functional nanoparticles or biomolecules is also highly desirable.

Other important challenge of 2D-hybrid nanomaterials-based electrochemical immunosensors is the real application. Although in almost every case very low LODs are achieved which are also better than those obtained with other approaches, most reports were performed with pure analytes in buffer solutions. Therefore, researchers should also make more effort on exploring the novel biosensor designs in complex matrices and solve the problem of nanomaterial aggregation in these media. Moreover, most reported graphene-based electrochemical immunosensors were designed and fabricated at lab-scale and are not suitable for commercial-scale production. Electrochemical biosensors need be designed and fabricated for commercial-scale production with good reproducibility and low cost.

Although accurate detection of various analytes has been achieved recently, the simultaneous detection of multiple targets in complex samples still remains unsolved and the possibility to controllably tune the nanomaterials' properties for simultaneous analysis of various analytes is yet to be explored. In this sense, it is critical to have a better understanding of $2 \mathrm{D}$-nanomaterials based 
on theoretical and experimental facts, as well as detection mechanisms and interactions between nanomaterials and varieties of interfaces, molecules, cells to design reliable and portable devices that would exhibit high performance such as the simultaneous detection of multiple targets with high selectivity and sensitivity. More choices of 2D-building blocks and their combinations are anticipated in the near future for further modulation of device properties and improvement of device performance. With the ability to prepare various 2D-dimensional-nanosized materials, combined with the advanced nanofabrication techniques, the current research in preparation of hybrid nanostructures lies on not only the choice of functional components, but also the spatial organization/assembly and geometric properties of the complex nanostructures. Furthermore, the integration of these 2D-nanohybrids based electrochemical biosensors into different analytical devices remains to be eye-caught in clinical point-of-care analysis.

In conclusion, although very promising, the field of 2D-nanomaterials hybrid immunosensors is still in its infancy and a number of challenging issues remain to be addressed. Fortunately, given its great potential, advances in this field, expected to be made rapidly, promise a better future for the biosensor industries. Moreover, maturation of this technology will allow for the high quality and large-scale fabrication of 2D-nanohybrid sensors while minimizing costs leading to its commercialization. It is expected also that future innovative research on this field will couple with other major technological advance, such as integration with realtime monitoring, lateral-flow, lab-on-chip, and 3D printing techniques for the development of next-generation biosensors.

\section{Acknowledgments}

The financial support of the Spanish Ministerio de Economía y Competitividad (CTQ2015-64402-C2-1-R) and the NANOAVANSENS Program from the Comunidad de Madrid (S2013/MT3029) Research Projects, are also gratefully acknowledged.

\section{Appendix A. Supplementary material}

Supplementary data associated with this article can be found in the online version at doi:10.1016/j.bios.2016.01.042.

\section{References}

Badhulika, S., Terse-Thakoor, T., Villarreal, C., Mulchandani, A., 2015. Front. Chem. 3, 38. http://dx.doi.org/10.3389/fchem.2015.00038.

Brownson, D.A.C., Banks, C.E., 2010. Analyst 135, 2768-2778.

Cai, Y., Li, H., Li, Y., Zhao, Y., Ma, H., Zhu, B., Xu, C., Wei, Q., Wu, D., Du, B., 2012 Biosens. Bioelectron. 36, 6-11.

Chen, H., Tang, D., Zhang, B., Liu, B., Cui, Y., Chen, G., 2012. Talanta 91, 95-102.

Cincotto, F.H., Martínez-García, G., Yañez-Sedeño, P., Canevari, T.C., Machado, S.A.S.,
Pingarrón, J.M., 2016. Talanta 147, 328-334.

Dharuman, V., Hahn, J.H., Jayakumar, K., Teng, W., 2013. Electrochim. Acta 114, 590-597.

Fang, Y.-S., Wang, H.-Y., Wang, L.-S., Wang, J.-F., 2014. Biosens. Bioelectron. 51, 310-316.

Feng, D., Lu, X., Dong, X., Ling, Y., Zhang, Y., 2013. Microchim. Acta 180, 767-774. Gan, X., Zhao, H., 2015. Sens. Mater. 27, 191-215.

Ge, S., Yu, F., Ge, L., Yan, M., Yu, V., Chen, V., 2012. Analyst 137, 4727-4733.

Gu, H., Zhou, T., Shi, G., 2015. Talanta 132, 871-876.

Han, J., Zhuo, Y., Chai, Y.-Q., Yuan, Y.-L., Yuan, R., 2012. Biosens. Bioelectron. 31 399-405.

Huang, X., Tan, C., Yin, Z., Zhang, H., 2014. Adv. Mater. 26, 2185-2204.

Huang, J., Tian, J., Zhao, Y., Zhao, S., 2015. Sens. Actuators B 206, 570-576.

Jia, X., Liu, Z., Liu, N., Ma, Z., 2014. Biosens. Bioelectron. 53, 160-166.

Jiang, S., Hua, E., Liang, M., Liu, B., Xie, G., 2013. Colloids Surf. B Biointerfaces 101, 481-486.

Kumar, N.A., Dar, M.A., Gul, R., Baek, J.-B., 2015. Mater. Today 18, 286-298.

Lee, J., Dak, P., Lee, Y., Park, H., Choi, W., Alam, M.A., Kim, S., 2014. Sci. Rep. 4, 7352. http://dx.doi.org/10.1038/srep07352.

Li, F., Gan, S., Han, D., Niu, L., 2015. Electroanal 27, 2098-2115.

Liu, Y., Liu, Y., Feng, H., Wu, Y., Joshi, L., Zeng, X., Li, J., 2012. Biosens. Bioelectron. 35, 63-68.

Liu, N., Ma, Z., 2014. Biosens. Bioelectron. 51, 184-190.

Liu, F., Xiang, G., Yuan, R., Chen, X., Luo, F., Jiang, D., Huang, S., Li, Y., Pu, X., 2014 Biosens. Bioelectron. 60, 210-217.

Lou, Y., He, T., Jiang, F., Shi, J.-J., Zhu, J.-J., 2014. Talanta 122, 135-139.

Lu, J., Liu, S., Ge, S., Yan, M., Yu, J., Hu, X., 2012. Biosens. Bioelectron. 33, 29-35.

Mao, H.Y., Laurent, S., Chen, W., Akhavan, O., Imani, M., Ashkarran, A.A., Mahmoudi, M., 2013. Chem. Rev. 113, 3407-3424.

Pumera, M., Loo, A.H., 2014. TrAC Trends Anal. Chem. 61, 49-53.

Samanman, S., Numnuam, A., Limbut, W., Kanatharana, P., Thavarungkul, P., 2015. Anal. Chim. Acta 853 (2015), 521-532.

Song, C., Xie, G., Wang, L., Liu, L., Tian, G., Xiang, H., 2014a. Biosens. Bioelectron. 58 68-74.

Song, H., Ni, Y., Kokot, S., 2014b. Biosens. Bioelectron. 56, 137-143.

Song, Y., Luo, Y., Zhu, C., Li, H., Du, D., Lin, Y., 2016. Biosens. Bioelectron. 76, 195-212.

Su, S., Chao, J., Pan, D., Wang, L., Fan, C., 2015. Electroanal 27, 1062-1072.

Sun, H., Chao, J., Zuo, X., Su, S., Liu, X., Yuwen, L., Fan, C., Wang, L., 2014. RSC Adv. 4, $27625-27629$

Sun, G., Zhang, L., Zhang, Y., Yang, H., Ma, C., Ge, S., Yan, M., Yu, J., Song, X., 2015. Biosens. Bioelectron. 71, 30-36.

Tang, J., Tang, D., Niessner, R., Chen, G., Knopp, D., 2011. Anal. Chem. 83, 5407-5414.

Tuteja, S.K., Sabherwal, P., Deep, A., Rastogi, R., Paul, A.K., Suri, C.R., 2014. ACS Appl. Mater. Interfaces 6, 14767-14771.

Wang, X., Chen, L., Su, X., Ai, S., 2013. Biosens. Bioelectron. 47, 171-177.

Wang, G., He, X., Chen, L., Zhu, Y., Zhang, X., 2014. Colloids Surf. B Biointerfaces 116 714-719.

Xia, X., Zheng, Z., Zhang, Y., Zhao, X., Wang, C., 2014. Sens. Actuators B 192, 42-50. Yagati, A.K., Pyun, J.-C., Min, J., Cho, S., 2016. Bioelectrochem 107, 37-44.

Yan, Z., Peng, Z., Casillas, G., Lin, J., Xiang, C., Zhou, H., Yang, Y., Ruan, G., Raji, O., A.R., Samuel, E.L.G., Hauge, R.H., Yacaman, M.J., Tour, J.M., 2014. ACS Nano 8 (5), 5061-5068.

Yang, G.-H., Shi, J.-J., Wang, S., Xiong, W.-W., Jiang, L.-P., Burda, C., Zhu, J.-J., 2013. Chem. Commun. 49, 10757-10759.

Yang, Z.-H., Zhuo, Y., Chai, Y.-Q., Yuan, R., 2014a. Sci. Rep. 4, 4747. http://dx.doi.org/ 10.1038/srep04747.

Yang, G.-H., Abulizi, A., Zhu, J.-J., 2014b. Ultrason. Sonochem. 21, 1958-1963.

Yang, G., Zhu, C., Du, D., Zhu, J., Lin., Y., 2015. Nanoscale 7, 14217-14231.

Yu, S., Wei, Q., Du, B., Wu, D., Li, H., Yan, L., Ma, H., Zhang, Y., 2013. Biosens. Bioelectron. 48, 224-229.

Yuan, G., Chen, H., Xia, C., Gao, L., Yu, C., 2015. Biosens. Bioelectron. 69, 95-99.

Zhang, S., Huang, N., Lu, Q., Liu, M., Li, H., Zhang, Y., Yao, S., 2015a. Biosens. Bioelectron. 77, 1078-1085.

Zhang, J., He, J., Xu, W., Gao, L., Guo, Y., Li, W., Yu, C., 2015b. Electrochim. Acta 156, 45-52.

Zhu, L., Xu, L., Jia, N., Huang, B., Tan, L., Yang, S., Yao, S., 2013. Talanta 116, 809-815.

Zhu, Q., Chai, Y., Zhuo, Y., Yuan, R., 2015. Biosens. Bioelectron. 68, 42-48. 